Published in "Chemical Physics Letters 574: 129-132, 2013"

which should be cited to refer to this work.

\title{
Electronic structure and magnetic properties of lanthanide 3+ cations
}

\author{
Helena Kurzen*, Laura Bovigny, Claudio Bulloni, Claude Daul \\ Department of Chemistry, University of Fribourg, Fribourg 1700, Switzerland
}

\begin{abstract}
The magnetic properties of lanthanides are currently of great interest. Especially, the discovery that mononuclear complexes of rare earth (RE) ions can exhibit magnetic anisotropy and can act as single molecular magnets. Heretofore, the magnetic susceptibility of RE ions has often been estimated, using HUND's empirical formula. In this work, we propose a non-empirical calculation of magnetic susceptibilities of Ln(III) ions based on Van Vleck's formula, that accounts for all excited states within the $\mathrm{f}^{n}$ manifold. The values obtained with Van Vleck's formula are much better than the ones with HUND's formula and are in good agreement with the experiments.
\end{abstract}

\section{Introduction}

Nowadays, it is impossible to imagine the world without lanthanides. They are important for many devices and technologies like single molecular magnets (SSM) or electronics (chip card, LED). Each day we use at least one object containing lanthanides, like the cellphone or the credit card. Since the beginning of the 19th there seems to be an endless love story between the rare earths (Y, Sc, Ln) and the lighting applications [1]. Seen that the lanthanides are systems with lots of electrons and the first group of atoms containing $f$ orbitals, they react different than the other groups.

A lot of experimental values of magnetic susceptibilities have been obtained for the lanthanides [2-5], but at the present time only few computed values are available. 35 years ago, a model has been developed for the computation of susceptibilities using the angular overlap approach [6] (AOM). The main goal of this Letter is to propose a reliable model for a simple calculation of magnetic susceptibilities of $\operatorname{Ln}($ III) free ions.

In this Letter we will calculate the energy levels (EL) of the trivalent lanthanide-ions with and without spin-orbit coupling. From the obtained results the magnetic behavior of each lanthanide-ion is calculated. For the calculations four MATLAB scripts have been written. One for the calculations without spin-orbit-coupling (SOC) and another one for the calculations including SOC. Before one of these calculations has been run, the corresponding parameters (Slater-Condon-parameters (SCP) and the SOC-constant zeta) had to be optimized. For the optimization a different script was written. The SCP are based on LF-DFT calculations and the SOCconstants are taken out of a collection of different values of different papers, compiled by Urland [7].

\footnotetext{
* Corresponding author.

E-mail address: helena.kurzen@unifr.ch (H. Kurzen).
}

\section{Calculation}

\subsection{Electronic structure of RE free ions}

The ground state electronic configuration of $\operatorname{Ln}(\mathrm{III})$ ions is [Xe] $\mathrm{f}^{n}$. The energy level structure of the $4 \mathrm{f}$ states is obtained from the following interactions, given in the decreasing order of their relative strength: the interelectronic repulsion, the spin-orbit coupling and the ligand field [8].

The Hamiltonian for a lanthanide free ion is

$H_{0}=-\frac{1}{2} \sum_{i=1}^{n} \nabla_{i}-\sum_{i=1}^{n} \frac{Z_{M}}{r_{i M}}+\sum_{i}^{n} \frac{1}{r_{i j}}+\sum_{i=1}^{n} \zeta_{i} \mathbf{l}_{i} \cdot \boldsymbol{s}_{i}$

with $Z_{M}$ the atomic number of $M, \boldsymbol{l}_{i}, \boldsymbol{s}_{i}$ the orbital and spin angular momenta of electron $i$, and $\zeta_{i}$ the spin-orbit coupling constant.

The spin-orbit coupling of RE ions is significantly stronger than in the case of $3 \mathrm{~d}$ transition metal ions. This is due to the larger nuclear charge of lanthanides. In the same time the ligand field is much lower, since the $4 \mathrm{f}$ orbitals are shrunk and $4 \mathrm{f}$ electrons are screened by the outer $5 \mathrm{~s}$ and $5 \mathrm{p}$ shells.

Under the approximation that spin-orbit coupling interaction is neglected, L and S are good quantum numbers. The Russell-Saunder coupling scheme describes well the energy levels structure of the ${ }^{2 S+1} L$ multiplets, where $L$ is the orbital and $S$ the spin orbital momentum [9].

When spin-orbit coupling contribution is included in the Hamiltonian, $L$ and $S$ are no longer good quantum numbers and the total angular momentum $J$ quantum number needs to be considered. The electronic structure which is observed is of $L_{j}^{2 S+1}$ multiplets. The ground state multiplet obeys HUND's rules and is $2 J+1$-fold degenerated.

The effect of the coordination of ligands on the energy levels of the free ion is the partial or complete removal of the $2 J+1$ degeneration inside the multiplets, due to the ligand field. The electronic 
ligand field Hamiltonian for a RE metal ion $\mathrm{M}$ surrounded by $\mathrm{N}$ ligands $L$, that is, a $\left[\mathrm{ML}_{N}\right]^{\mathrm{q}}$ complex reads

$H_{L F}=H_{0}-\sum_{i=1}^{n} \sum_{i=1}^{N} \frac{Z_{L}^{\prime}}{r_{i i}}$

with $Z_{L}^{\prime}$ the effective charge of ligand $L$ and $r_{i i}$ the distance between electron $i$ and ligand 1 . The expression above differs from the Hamiltonian for the free ion the ligand field potential

$V_{L F}=-\sum_{i=1}^{n} \sum_{i=1}^{N} \frac{Z_{L}^{\prime}}{r_{i i}}$

which accounts for the electrostatic field generated by the $N$ ligands on the $n \mathrm{f}$ electrons of RE ion. In the ligand field model, the perturbation $V_{L F}$ is calculated as a sum of adjustable parameters, the socalled ligand field parameters

$V_{L F}=\sum_{k=0}^{2 i} \sum_{q=-k}^{+k} h_{k q} Y_{k q}$

with $Y_{k q}$ the spherical harmonics and $h_{k q}$ the ligand field parameters.

\subsection{Magnetic susceptibility}

The main property which has been determined in this work is the magnetic volume susceptibility $\chi$ of the $\operatorname{Ln}(\mathrm{III})$ series, from $\mathrm{Pr}$ to Tm.

The determination of magnetic susceptibility involves the consideration of Zeeman interaction of the Ln(III) free ion system. This perturbation arises when a magnetic field $\boldsymbol{H}$ is applied and is equal to

\section{$\boldsymbol{H}_{\text {Zeeman }}=\boldsymbol{\mu} \cdot \boldsymbol{H}$}

with $\boldsymbol{\mu}$ the magnetic moment

$\boldsymbol{\mu}=\mu_{B}\left(\boldsymbol{L}+g_{e} \boldsymbol{S}\right)$

with $\mu_{B}$ the Bohr magneton, $g_{e}$ the gyromagnetic ratio of the free electron and $\boldsymbol{L}, \boldsymbol{S}$ are the orbital and spin angular momenta respectively. The Zeeman perturbation has to be included in the full electronic Hamiltonian when magnetic susceptibility measures are performed.

The aim of our calculations was to compare the values of the effective Bohr magneton number $\mu_{\text {eff }}$ calculated by means of Van Vleck formula and from empirical HUND's formula which is known to fit well with observed magnetic moments for all $\operatorname{Ln}(\mathrm{III})$ but $\mathrm{Sm}(\mathrm{III})$ and $\mathrm{Eu}(\mathrm{III})$ [10-12].

Van Vleck formula describes the dependence of magnetic susceptibility on temperature, and is one of the cornerstone relations in magnetochemistry.

$\chi(T)=\frac{N \sum_{n}\left[\frac{\left(E_{n}^{(1)}\right)^{2}}{k T}-2 E_{n}^{(2)}\right] \exp \left(-\frac{E_{n}^{(0)}}{k T}\right)}{\sum_{n} \exp \left(-\frac{E_{n}^{(0)}}{k T}\right)}$

The effective Bohr magneton number (commonly defined as the effective magnetic moment) $\mu_{e f f}$ is usually deduced from the magnetic susceptibility thanks to the relation

$\mu_{e f f}=\left(\frac{3 k_{B} \chi^{T}}{\mu_{0} N_{A} \mu_{B}^{2}}\right)^{1 / 2}$

HUND's rule originates from a simplified model which assumes that the magnetic moments of $\mathrm{Ln}$ is well described from the coupling of the spin and orbital angular momenta, i.e. the RussellSaunders LS coupling scheme yielding a total angular momentum $J$ defined as
$J=|L-S|,|L-S+1|, \ldots,|L+S|$

The main assumptions of this model are that only the ground $J$ state is populated and that the ligand field splitting is very small compared to the spin orbit coupling effects so that the magnetism is essentially independent of the chemical environment.

The expression of the magnetic moment $\mu_{J}$ in terms of the total angular momentum quantum number $J$ is the HUND's rule

$\mu=g_{J} \sqrt{J(J+1)} \mu_{B}$

with the Landé g-factor $g_{J}$ defined as

$g_{J}=1+\frac{J(J+1)+S(S+1)-L(L+1)}{2 J(J+1)}$

The theoretical values of the magnetic moments of $\mathrm{Ln}^{3+}$ calculated with HUND's formula are generally in good agreement with the experimental magnetic moments, with the exception of $\mathrm{Sm}^{3+}$ and $\mathrm{Eu}^{3+}$ which will be considered in more detail in the discussion of the results.

\subsection{Ab initio calculation of Slater-Condon parameters by LF-DFT method}

First principle calculation of Slater-Condon parameters $F_{0}, F_{2}, F_{4}$ and $F_{6}$ for the $\operatorname{Pr}(\mathrm{III})-\mathrm{Gd}(\mathrm{III})$ series was performed using LF-DFT method using the ADF 2010 software package. MATLAB scripts were written in order to generate all possible microstates (Slater determinants) for each $\mathrm{f}$ configuration (from $\mathrm{f}^{2}$ up to $\mathrm{f}^{7}$ ) and to determine the energies of the terms when only the interelectronic repulsion is considered as a perturbation. In the following the lanthanide pairs $\mathrm{Pr} / \mathrm{Tm}, \mathrm{Nd} / \mathrm{Er}, \mathrm{Pm} / \mathrm{Ho}, \mathrm{Sm} / \mathrm{Dy}$ and $\mathrm{Eu} / \mathrm{Tb}$ are considered as isoelectric. The $F_{0}$ parameter has been set at the origin of the energy scale and the hole-electron equivalence was exploited when considering the computation of the parameters for the $\mathrm{f}^{7}-$ $\mathrm{f}^{14}$ electronic configurations.

The parameters calculated by DFT were taken as the starting values for the optimization in which the difference between the experimental and theoretical energies of the terms arising from $\mathrm{f}^{2}-\mathrm{f}^{14}$ configurations were minimized by variation of the reduced matrix elements of the atomic interelectronic repulsion for $\mathrm{f} \mathrm{elec-}$ trons. The expected sources of error which has to be taken into account when computing these reduced matrix elements with ADF package are that the reference for the energy is the neutral atom and that the $f$ orbitals are not well defined.

\subsection{Parameter optimization}

To avoid to imprecise values, the SCP and the zeta-parameters were optimized for each lanthanide. With small iteration steps the values were changed in order to minimize

lsq $=\operatorname{norm}\left(E_{\text {theory }}-E_{\text {experimental }}\right)$

For the error calculation the calculated energies were compared with the experimental ones from NIST. During the optimization the values were compared and the parameters were adjusted just until the error became smaller than $10^{-8}$. The optimized values were checked each time to make sure, to really get optimized values. The starting values for the zeta optimization were found in Refs. [12-19].

\section{Results and discussion}

There is a need of correct energies for a good calculation of the magnetic susceptibility. So there is a big interest that the calculated energy levels (EL) are as close as possible to the experimental values. Therefore optimizations were carried out for each lantha- 
Table 1

SCP by LF-DFT calculation and zeta by references [14-21] and both optimized by least squares energy minimization of each lanthanide-ion, except the zeta of Gd ${ }^{3+}$. The theoretical SCP found in [22] are divided by 5, 9 and 13 , respectively in order to allow comparison with our results.

\begin{tabular}{|c|c|c|c|c|c|c|}
\hline $\mathrm{Ln}^{3+}$ & $F_{0}$ & $F_{2}$ & $F_{4}$ & $F_{6}$ & $\zeta$ & Ref. \\
\hline $\mathrm{Pr}^{3+}$ & 0 & $14002(13776)$ & $6426(5594)$ & $2552(2531)$ & $729.50(729.5)$ & {$[14,22]$} \\
\hline $\mathrm{Nd}^{3+}$ & 0 & 13989 (14604) & 7019 (5865) & $504(2751)$ & $719.12(884.58)$ & {$[15,22]$} \\
\hline $\mathrm{Pm}^{3+}$ & 0 & 21118 & 5301 & 1030 & 644.21 & \\
\hline $\mathrm{Sm}^{3+}$ & 0 & 11612 & 9794 & 2048 & $1625.10(1420)$ & [16] \\
\hline $\mathrm{Eu}^{3+}$ & 0 & 12829 & 22280 & 620 & $2122.91(1320)$ & [17] \\
\hline $\mathrm{Gd}^{3+}$ & 0 & 17280 & 11107 & 816 & $1598.50(1450)$ & [18] \\
\hline $\mathrm{Tb}^{3+}$ & 0 & 15187 (17799) & 18244 (6991) & $1376(3635)$ & 2201.29 (1705) & {$[17,22]$} \\
\hline $\mathrm{Ho}^{3+}$ & 0 & 17423 (18913) & $5078(7377)$ & $392(4002)$ & $3342.00(2163)$ & {$[19,22]$} \\
\hline $\mathrm{Er}^{3+}$ & 0 & 25639 (19497) & $2066(7545)$ & $1163(4155)$ & $2809.49(2393.34)$ & {$[20,22]$} \\
\hline $\mathrm{Tm}^{3+}$ & 0 & 42506 & 7587 & 158 & $2571.31(2617)$ & {$[21]$} \\
\hline
\end{tabular}

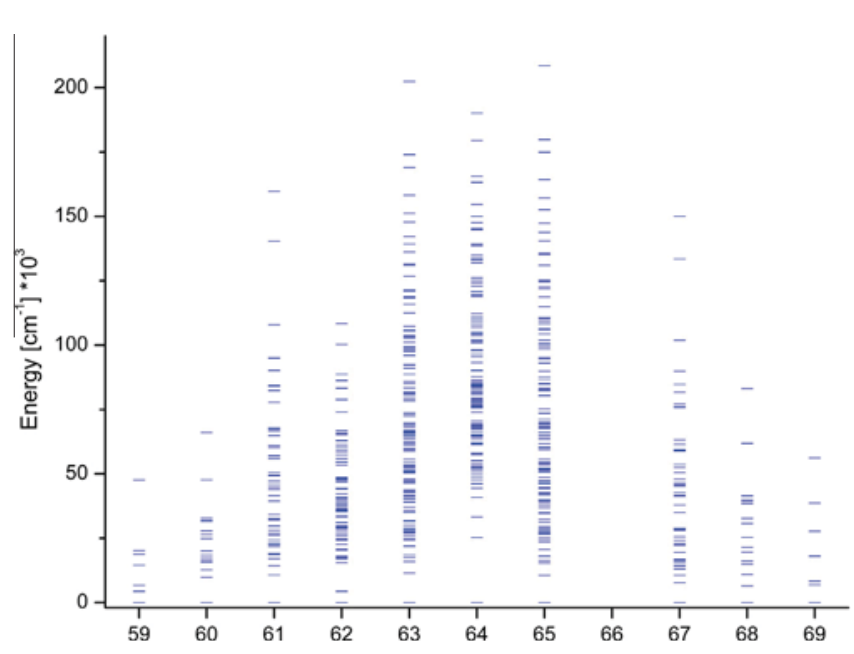

Figure 1. Energy levels without SO-coupling by their atomic number.

nide-ion. In a first step the SCP were optimized to get optimal EL without SOC, and in a second step the optimized SCP were used for the optimization of the zeta-parameter to get the EL with SOC. Seen that there are only two experimental values for the EL of the dysprosium-ion (No. 66) the results are not significant and therefore not considered in Table 1, Figures 1 and 3.

In Table 1 the final optimized SC- and zeta-parameters are represented. Remarkable is the surge from $\mathrm{Pm}^{3+}$ to $\mathrm{Sm}^{3+}$. The value of zeta rises to about 1000 . The general trend of the raising values can be explained with the rising number of valence electrons.

Figure 1 represents the result of the first part of our calculations. The EL of each lanthanide-ion is illustrated by taking the energy states vs. atomic number. To show the distribution of the different states, in Figure 2 the $\mathrm{f}^{2}, \mathrm{f}^{3}$ and $\mathrm{f}^{4}$ configurations were assigned and compared.

The visible symmetry of the results resented in Figure 2 agrees with the previous isoelectric considerations. Moreover the assignments of each configuration are in agreement with each other. The procedure and the result would be the same for the other configurations. But the number of states increases and with this the states get closer. So it is impossible to present them in an easy way.

In the second part the spin-orbit coupling was taken in account. The energy levels from the orbit-was first step have split into several degenerated levels. The resulting ELs are presented in Figure 3. These energies were used for the calculation of the magnetic susceptibility.

Due to the big amount of ELs it is not possible anymore to assign the states directly. To illustrate hole-electron equivalence, the ELs of the $\mathrm{f}^{2}$ configurations are compared in Table 2 . The isoelectricity is the highest for $\mathrm{f}^{2}$ configurations. The description of the holeelectron equivalence for $\mathrm{f}^{n}$ states with $n>2$ is difficult to recognize the symmetry, because the assignment gets de more and more subtle. The ELs without SOC are already close to each other. With the degeneracy they are not only close to each other but also mixed. So the states cannot be assigned with good level of significance.

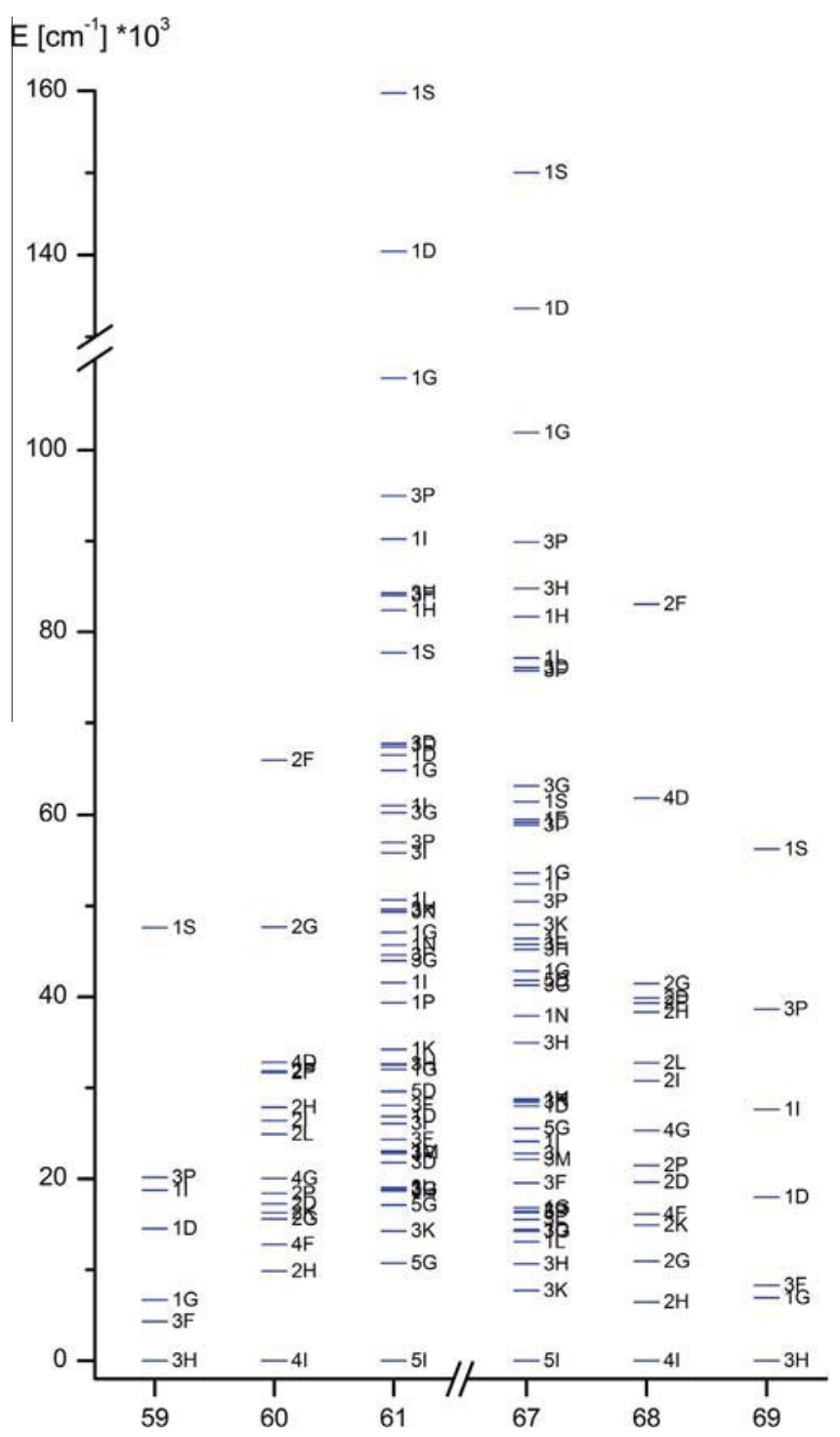

Figure 2. Energy levels without SO-coupling, $\mathrm{f}^{2}, \mathrm{f}^{3}, \mathrm{f}^{4}$. 


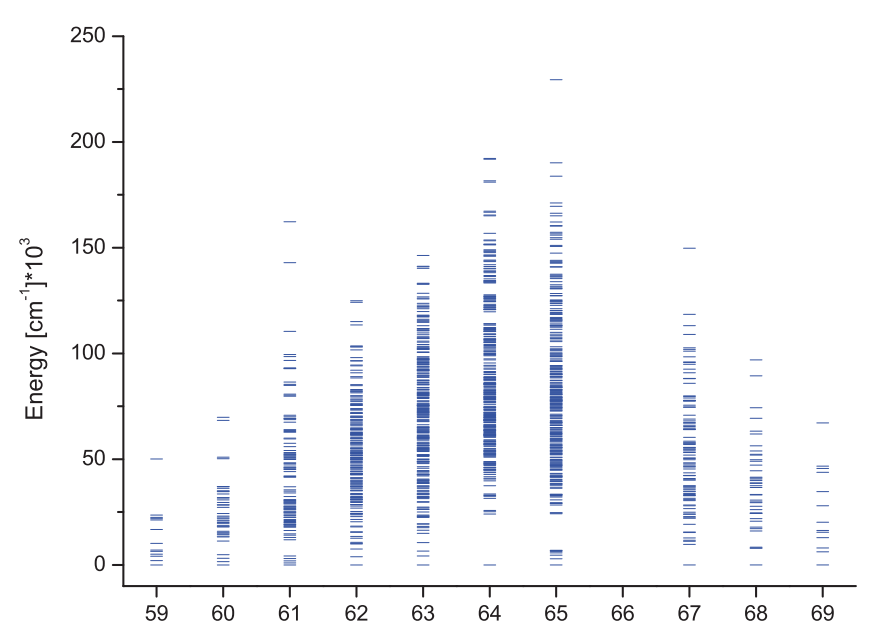

Figure 3. Energy levels with SO-coupling by their atomic number.

Table 2

Energy splitting of $\mathrm{f}^{2}$ Lanthanides(III).

\begin{tabular}{|c|c|c|c|c|c|}
\hline \multicolumn{6}{|l|}{ f2 } \\
\hline \multicolumn{3}{|c|}{$\operatorname{Pr}^{3+}$} & \multicolumn{3}{|c|}{$\mathrm{Tm}^{3+}$} \\
\hline \multirow[t]{3}{*}{$3 \mathrm{H}$} & 4 & 0.0 & $3 \mathrm{H}$ & 6 & 0.0 \\
\hline & 5 & 2024.0 & & 5 & 8002.7 \\
\hline & 6 & 4165.7 & & 4 & $12,904.9$ \\
\hline \multirow[t]{3}{*}{$3 F$} & 2 & 5048.5 & $3 \mathrm{~F}$ & 4 & $61,25.5$ \\
\hline & 3 & 6417.2 & & 3 & $16,282.1$ \\
\hline & 4 & 7037.4 & & 2 & $27,954.6$ \\
\hline $1 \mathrm{G}$ & 4 & $10,143.2$ & 1D & 2 & $15,370.1$ \\
\hline 1D & 2 & $16,724.9$ & $1 G$ & 4 & $20,154.1$ \\
\hline 1I & 6 & $21,244.0$ & $1 \mathrm{I}$ & 6 & $34,629.5$ \\
\hline \multirow[t]{3}{*}{$3 P$} & 0 & $21,932.2$ & $3 P$ & 0 & $43,784.1$ \\
\hline & 1 & $22,525.6$ & & 2 & $45,608.0$ \\
\hline & 2 & $23,616.3$ & & 1 & $46,639.7$ \\
\hline $1 \mathrm{~S}$ & 0 & $50,084.6$ & $1 \mathrm{~S}$ & 0 & $67,083.9$ \\
\hline
\end{tabular}

\subsection{Magnetic susceptibility}

In the last part the magnetic susceptibility, represented in Figure 4, was calculated. But first a region of acceptable values was determined according to experimental values [2-5]. Further the

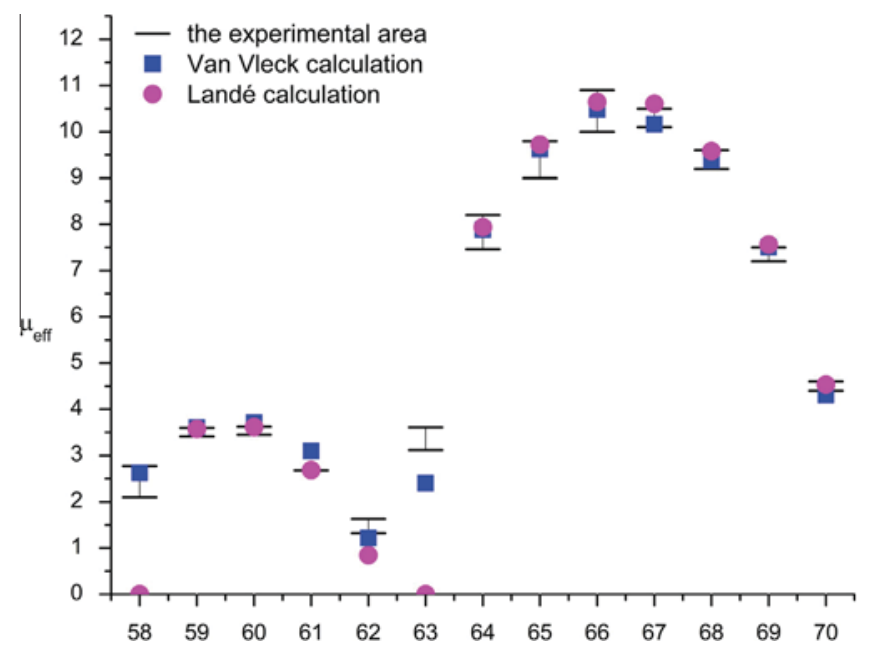

Figure 4. Magnetic susceptibility of $\operatorname{Ln}^{3+}$ (with the atomic numbers) at room temperature. magnetic susceptibility was calculated by HUND's rule, which takes only in account the ground state. The HUND's calculation for the magnetic susceptibilities is in agreement to the experiments, except for $\mathrm{Sm}^{3+}$ and $\mathrm{Eu}^{3+}$ where its experimental value are too large. In a publication written by Laporte (1928) [13], this anomaly has been supposed to be related to the small energy difference between the ground and first excited multiplet, which is close to $k \mathrm{~T}$. This situation is more pronounced in $\mathrm{Eu}^{3+}$ and to a lesser extent in $\mathrm{Sm}^{3+}$. Moreover the values of $\mathrm{Dy}^{3+}$ are very close to the experimental ones, although the results of the EL calculation were not significant.

To get smaller errors, the procedure was repeated with the Van Vleck formula. This formula also considers the excited states and not only the ground state. Even if the Van Vleck formula there are still some errors for $\mathrm{Eu}^{3+}$ and $\mathrm{Tb}^{3+}$. This deviation could probably be explained, supposing that the experimental values stem from measurements of complexes and not of free ions.

\section{Conclusion}

In this Letter we show that the calculated magnetic susceptibility of free ions differs not a lot to the measurements of the complexed lanthanides. This is in agreement to our simplification not to consider the ligand field.

A simple semi-empirical formula, HUND, can approximate the magnetic susceptibility for a majority of the lanthanides, but to get better results, the Van Vleck formula should be used instead.

Moreover we calculated the different ELs of each Lanthanide with and without SOC and assigned most of the levels.

The optimized Slater-Condon parameters, calculated by LF-DFT and further optimizations, agree well with the measured ones.

\section{Acknowledgements}

We want to thank Andrea Barbiero (University of Fribourg) for his help during this project. The authors are grateful to Werner Urland (University of Hannover) for discussions on magnetism and rare earths. Special words of gratitude to Harimbola Ramanantoanina (University of Fribourg) for his help with the DFT calculations and his support during the whole project.

This work was supported by the Swiss National Science Foundation.

\section{References}

[1] S.V. Eliseeva, J.-C.G. Bünzli, New J. Chem. 35 (2011) 1165.

[2] B. Cabrera, Comptes Rendus (1925) 668

[3] S. Meyer, Physical Zeits (1925) 478.

[4] H. Deeker, Ann. Der Phys. (1926) 324.

[5] J. Zernike, C. James, J. Am. Chem. Soc. 48 (1926) 2827

[6] W. Urland, Chem. Phys. Lett. 46 (1976) 393.

[7] Urland, P. D. W. Homepage des Arbeitskreises http://www.unics.unihannover.de/nhccurla/chemie/index_chemie.html (accessed Feb 11, 2012).

[8] J. Luzon, R. Sessoli, Dalton Trans. 41 (2012) 13556.

[9] L. Sorace, C. Benelli, D. Gatteschi, Chem. Soc. Rev. 40 (2011) 3092.

[10] S. Hatscher, H. Schilder, H. Lueken, W. Urland, Pure Appl. Chem. 77 (2005) 497.

[11] Van Vleck, J.H. The Theory of Electric and Magnetic Susceptibilities; Fowler, R. Kapitza, P., Eds., Oxford University Press, Oxford, 1932.

[12] H. Lueken, Magnetochemie: Eine Einführung in Theorie und Anwendung, Vieweg+teubner Verlag, 1999.

[13] O. Laporte, Z. Phys. 47 (1928) 761

[14] J.S. Margolis, J. Chem. Phys. 35 (1961) 1367.

[15] J.C. Eisenstein, J. Chem. Phys. 39 (1963) 2134.

[16] W. Urland, Ber. Bunsenges. Phys. Chem. 85 (1981) 1139

[17] F. Soetebier, Dissertation, Univ. Hannover, 2002.

[18] C.-G. Ma, M.G. Brik, W. Ryba-Romanowski, H. Swart, M.A. Gusowsky, J. Phys. Chem. (2012) 9158.

[19] Croizier, Runciman, J. Chem. Phys. 35 (1961) 1392.

[20] J.C. Eisenstein, J. Chem. Phys. 39 (1963) 2128.

[21] Gruber, Krupke, Poindexter, J. Chem. Phys. 83 (1979) 1042.

[22] D.J. Newman, B. Ng, Crystal Field Handbook, Cambridge University Press, 2007. 